\author{
B. M. H. Lange · G. Kirfel · I. Gestmann · V. Herzog \\ C. González
}

\title{
Structure and microtubule-nucleation activity of isolated Drosophila embryo centrosomes characterized by whole mount scanning and transmission electron microscopy
}

Accepted: 5 July 2005/Published online: 10 August 2005

(C) Springer-Verlag 2005

\begin{abstract}
Experimental approaches in Drosophila melanogaster over the last 20 years have played a fundamental role in elucidating the function, structure and molecular composition of the centrosome. However, quantitative data on the structure and function of the Drosophila centrosome are still lacking. This study uses, for the first time, whole mount electron microscopy in combination with negative staining on isolated centrosomes from the early Drosophila embryos to analyze its dimensions, structure and capacity to nucleate microtubules in vitro. We show that these organelles are on average $0.75 \mu \mathrm{m}$ in diameter and have abundant pericentriolar material which often appears fibrillar and with bulbous protrusions. Corresponding to the abundant pericentriolar material, extensive microtubule nucleation occurs. Quantification of the number of microtubules nucleated showed that 50-300 active nucleation sites are present. We examined via electron microscopy immunogold labeling the distribution of $\gamma$-tubulin, CNN, Asp and the MPM-2 epitopes that are phosphorylated through Polo and the Cdk1 kinase. The distribution of these proteins is homogeneous, with the MPM-2 epitopes exhibiting the highest density. In contrast, centrosomal subdomains are identified using a centriole marker to relate centrosome size to the centriole number by electron microscopy. In conclusion, we present a clear-cut technique assaying and quantifying the
\end{abstract}

\footnotetext{
B. M. H. Lange $(\bowtie)$

Max-Planck Institute for Molecular Genetics,

Vertebrate Genomics, Ihnestr. 73,

14195 Berlin, Germany

E-mail: lange_b@molgen.mpg.de

Tel.: + 49-30-84131645

Fax: +49-30-84131128

G. Kirfel · I. Gestmann · V. Herzog

Institute for Cell Biology, Ulrich-Haberland-Strasse 61a, 53121 Bonn, Germany

C. González

ICREA and IRBB, Parc Científic de Barcelona,

C/Josep Samitier 1-5,

08028 Barcelona, Spain
}

microtubule nucleation capacity and antigen distribution complementing molecular studies on centrosome protein complexes, cell organelle assembly and protein composition.

Keywords Centrosome $\cdot$ Drosophila $\cdot$ Microtubule MTOC $\cdot$ SEM $\cdot$ TEM $\cdot$ Whole-mount preparation

\section{Introduction}

The centrosome is the main microtubule-organizing center (MTOC) in higher eukaryotic cells (for a recent review see Doxsey 2001). It is defined as a pair of centrioles surrounded by pericentriolar material that nucleates a radial array of microtubules playing a role in maintaining cell shape, in organelle transport and in mitosis. Many excellent electron microscopic studies investigated this organelle in a variety of different organisms (reviewed in Bornens 2002; Gonzalez et al. 1998; Kellogg et al. 1994; Vorobjev and Nadezhdina 1987) and defined details of centriole and centrosome structure. Most of this work though, focuses on the centrioles, the most conspicuous structures in the centrosome, comprising a pair of cylinder-like structures composed of microtubule triplets arranged with 9-fold radial symmetry (Lange and Gull 1996a; Marshall 2001). The centriole is the template for the axoneme structure in ciliated or flagellated cells, but has also been suggested to play a role as congregation site for pericentriolar material and in controlling the structural stability of the centrosome (Bornens 2002). In contrast, the pericentriolar material is structurally less well defined and has often been described as "amorphous" or "electron dense" material. Recently however, electron microscopy tomography and high-resolution fluorescence microscopy of centrosomes from Drosophila, Spisula and mammalian cells have identified ring-like structures and protein complexes of higher order (Dictenberg et al. 1998; Moritz et al. 1995a; Vogel 
et al. 1997). These ring-like structures colocalize with gamma-tubulin, a conserved centrosomal component, in immunoelectron microscopy (Moritz et al. 1995b) suggesting them to be akin to the $\gamma$-tubulin ring complex $(\gamma-$ TuRC) involved in microtubule nucleation.

The $\gamma$-TuRCs, the functional units of the centrosome, localize to the proximal end of microtubules acting both as capping and microtubule nucleation site involved in blocking microtubule assembly and disassembly (Keating and Borisy 2000; Moritz et al. 2000; Wiese and Zheng 2000). Structurally, $\gamma$-tubulin and the other proteins of the $\gamma$-TuRC are linked through $\gamma$-tubulin-binding proteins to the centrosomal scaffold, a proteinaceous matrix maintaining a coherent centrosome organization (Moritz et al. 1998; Schnackenberg et al. 1998).

While some of the molecular mechanisms of microtubule nucleation have been revealed, we are still missing data on the distribution of different centrosomal proteins in the centrosome at an electron microscopic level as well as quantitative data on the number of $\gamma$-TuRCs in the centrosome. Such information would indicate how many molecular complexes are actually involved in the process of microtubule nucleation. Moreover, it would complement ongoing studies elucidating the precise molecular composition of the centrosome. Experimental approaches in Drosophila melanogaster have played a fundamental role in elucidating the function, structure and molecular composition of the centrosome (reviewed by Gonzalez et al. 1998; Kellogg et al. 1994; Rothwell and Sullivan 2000). However, despite the long history of research on the Drosophila centrosome, we still lack quantitative data on its structure and its capacity to nucleate microtubules. To obtain such information we isolated centrosomes from Drosophila early embryos and analyzed them using a whole mount technique in combination with either transmission or scanning electron microscopy.

Our results indicate that within a population of isolated centrosomes, organelle size is varying twofold while the number of nucleated microtubules varies about six times between the small and large asters. This variation in centrosome size and hence both centrosome surface and number of nucleated microtubules correlates well. Furthermore, we investigated the distribution of $\gamma$ tubulin, CNN, Asp and of MPM-2 phosphoepitopes, at the centrosome. These proteins have been suggested either to play a structural and functional role for microtubule nucleation or in the case of the MPM-2 phosphoepitope that are substrates of cell cycle regulatory kinases. In our whole mount assay, antigen distribution on the centrosome was homogeneous. In contrast a centriolar marker, hence labeling a structural subdomain of the centrosome, allows us to correlate centrosome size to centriole number in the centrosome.

Taken these data together, we provide new quantitative data on isolated centrosomes from the early Drosophila embryo that is on average $0.75 \mu \mathrm{m}$ in diam- eter and contains between 50 and 300 active microtubule nucleation sites.

\section{Methods}

\section{Centrosome preparation}

Centrosomes were prepared from early Drosophila embryos (0-3 1/2-h old) according to Moritz et al. (1995a). Sucrose fractions were assayed for centrosomes by immunofluorescence microscopy with the anti- $\gamma$-tubulin antibody GTU-88. Peak fractions were aliquoted, frozen in liquid nitrogen and stored at $-80^{\circ} \mathrm{C}$. The centrosomes were strongly enriched but impurities of chromatin were detected by fluorescence light microscopy and electron microscopy examination. For this reason only centrosomes that were unequivocally identified by immunogold labeling electron microscopy were measured.

Whole mount preparation of centrosomes onto glass coverslips and EM grids

Typically, $20 \mu \mathrm{l}$ of a centrosome containing fraction were diluted in $9 \mathrm{ml}$ of phosphate buffered saline (PBS) and spun down onto $11 \mathrm{~mm}$ round glass cover slips at $12,000 \mathrm{rpm}$ in a Sorvall HB4 rotor for $15 \mathrm{~min}$ at $4^{\circ} \mathrm{C}$. The glass slides and EM grids (Plano, Wetzlar, Germany) were supported by custom made plastic inserts for Corex glass tubes, according to Evans et al. (1985). The formvar and carbon-coated EM grids were attached laterally to a piece of double sided sticky tape on the plastic support.

\section{Microtubule aster nucleation assay}

Microtubule nucleation was performed according to Evans et al. (1985). An amount of $20 \mu \mathrm{l}$ of centrosomescontaining sucrose fraction were incubated with phosphocellulose purified tubulin at $2.5 \mathrm{mg} / \mathrm{ml}$ (saturating concentration). Asters were fixed in solution with $0.75 \%$ glutaraldehyde in BRB80 buffer (80 mM PIPES, $1 \mathrm{mM}$ $\mathrm{MgCl}_{2}, 1 \mathrm{mM}$ EGTA, pH 6.8), spun through a $40 \%$ glycerol cushion either onto a $11 \mathrm{~mm}$ round glass coverslip or onto freshly glow-discharged EM grids in a Sorvall HB4 rotor for $15 \mathrm{~min}$ at $12,000 \mathrm{rpm}$ at $20^{\circ} \mathrm{C}$ and then processed for immunofluorescence or electron microscopy, respectively as described below in sections 2.5-2.7.

\section{Immunofluorescence microscopy}

Samples prepared as described in Sections 2.2 and 2.3 were subsequently removed from the Corex glass tubes 
and fixed for at least $5 \mathrm{~min}$ in methanol at $-20^{\circ} \mathrm{C}$. They were rehydrated in PBS for $15 \mathrm{~min}$ and immunostaining was performed according to MacRae et al. (1990).

Transmission EM preparation and analysis

Contrasting was performed with the negative stain goldthioglucose (Sigma, Munich, Germany) according to Lange and Gull (1996b) with the difference that centrosomes were spun down onto EM grids (see Section 2.2) rather than pelleted in a Beckman airfuge rotor. The samples were inverted onto a drop of $3.7 \%$ paraformaldehyde in BRB80 buffer and fixed for $30 \mathrm{~min}$ at room temperature, then washed twice by floating on a drop of PBS and finally stained. Samples were viewed in a Philips 400 transmission electron microscope at low beam intensities to avoid stain precipitation. Measurements were carried out for each centrosome twice in two different diagonals and then averaged.

\section{Immunogold labeling}

Samples prepared as described in Sections 2.2 and 2.3, were fixed in $3.7 \%$ paraformaldehyde in BRB80 buffer for $30 \mathrm{~min}$ at room temperature. Samples were washed three times with PBS and then free binding sites were blocked with sterile filtered $1 \%$ BSA in PBS for $1 \mathrm{~h}$. First and second antibody incubations were performed for $1 \mathrm{~h}$ both followed by four washing steps. All incubation and washing steps were performed in $0.1 \%$ BSA in PBS. The final washing step was in PBS only.

\section{Scanning electron microscopy (SEM)}

EM grids with isolated centrosomes (see above) were mounted on aluminum sample holders and sputter coated with $2 \mathrm{~nm}$ platinum/palladium in a HR 208 coating device (Cressington, Watford, UK). SEM was performed at an acceleration voltage of $3 \mathrm{kV}$ using an XL 30 SFEG (Philips, Eindhoven, The Netherlands) equipped with a through lens secondary electron detector.

Dephosphorylation of centrosomal phospho-protein epitopes

Centrosomes were spun down onto EM grids as described above and then floated at room temperature for $20 \mathrm{~min}$ on a drop of phosphatase buffer $(10 \mathrm{mM} \mathrm{NaCl}$, $5 \mathrm{mM}$ Tris- $\mathrm{HCl}, 1 \mathrm{mM} \mathrm{MgCl} 2,1 \mathrm{mM}$ dithiothreitol, $\mathrm{pH}$ 7.9) containing $30 \mathrm{U} / \mathrm{ml}$ alkaline phosphatase (New England Biolabs, Frankfurt) and $2.5 \mathrm{mM}$ PMSF. Samples were washed three times by floating the grids on blocking buffer and then prepared for immunogold labeling and negative staining as described above.
Antibodies used

The following antibodies were used. The anti-alphatubulin DM1A and anti- $\gamma$-tubulin GTU88 antibodies were obtained from Sigma (Munich, Germany). The anti-CNN antibody ( $\mathrm{Li}$ and Kaufman 1996), and the anti-asp antibody (Saunders et al. 1997) were kind gifts of Drs. Kaufman (Bloomington, USA) and Glover (Cambridge, UK), respectively. The MPM2 antibody was purchased from Biomol (Hamburg, Germany). Secondary antibodies, gold conjugated $(10 \mathrm{~nm})$ antibodies were obtained from Plano (Wetzlar, Germany). The secondary Alexa 488-conjugated anti-mouse antibody was from Molecular Probes (Leiden, The Netherlands).

\section{Results}

Isolation and whole mount negative staining of Drosophila early embryo centrosomes

First, we carried out a basic structural characterization of the Drosophila centrosomes by whole mount preparation, a technique which is very informative in determining overall shape, size distribution, antigen localization and surface contour. Centrosomes were isolated according to Moritz et al. (1995a) by sucrose gradient centrifugation. Sucrose fractions were assayed by spinning small aliquots of each sample down onto glass slides (Evans et al. 1985). Then samples were fixed and processed for immunofluorescence microscopy with an anti- $\gamma$-tubulin monoclonal antibody and assayed for the peak fractions containing the highest numbers of centrosomes. Centrosomes were isolated structurally intact as well as were competent in microtubule nucleation as judged by immunofluorescence and electron microscopy, indicating that fully functional organelles were purified.

Centrosomes labeled for immunofluorescence microscopy with anti- $\gamma$-tubulin antibodies revealed a distribution of varying sizes (Fig. 1a). Since the expected centrosome size was only about two to three times the limit of resolution of the light microscope we did not consider a quantitative analysis of the size distribution by light microscopy useful. Hence, we decided to proceed with a structural and immunological analysis by electron microscopy.

Selected fractions were further analyzed by pelleting centrosomes onto electron microscopic grids. Formaldehyde fixed samples were contrasted with Goldthioglucose and viewed by transmission electron microscopy. Whole mount preparations of negatively contrasted samples showed that the Drosophila centrosomes have a sphere-like shape with abundant pericentriolar material that rarely allows detection of the centriole (Fig. 1b). Their appearance was overall uniform, centrosomes looked intact with a surface contour 


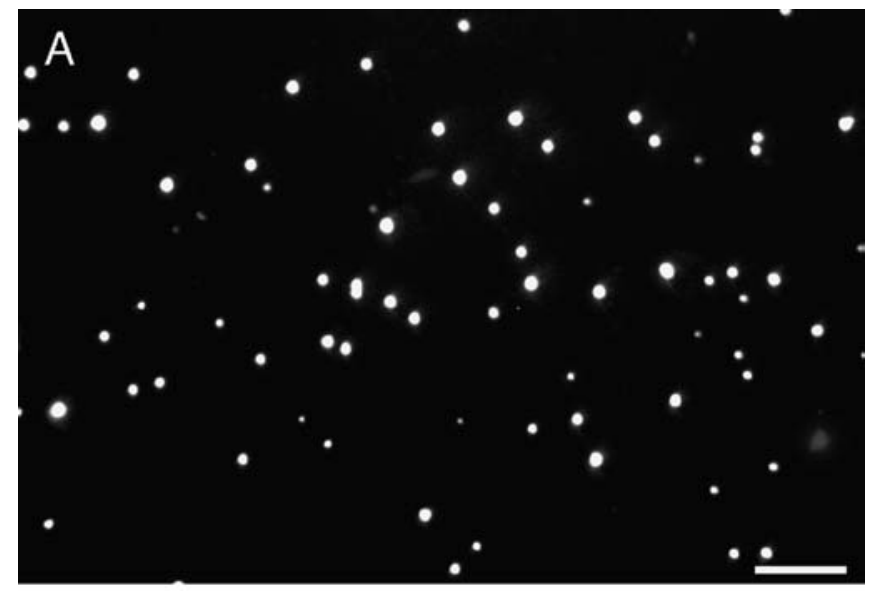

B

Fig. 1 Overview of centrosome preparations isolated from the syncytial stage of Drosophila embryos. a Immunofluorescence microscopy micrograph of centrosomes labeled with an antigamma-tubulin antibody to identify centrosomes in sucrose gradient fractions. The centrosomes vary in size but no other morphological features can be discerned $($ bar $=7 \mu \mathrm{m})$. b TEM micrograph of whole-mount preparation of Drosophila centrosomes, contrasted with aurothioglucose, revealing their round shape with the rough fibrillar surface structure of the pericentriolar material $(\mathrm{bar}=0.5 \mu \mathrm{m})$

that always appeared rough and structured. Often the surface appeared slightly segmented and fibrillar, sometimes with protruding bulbous pieces of pericentriolar material (Figs. 1b and 3).

Size determination of immunogold labeled centrosomes

We examined immunogold labeled centrosomes $(n=162)$ to obtain an overview of the size distribution and strucutre in the population of the isolated organelles. Quantification was performed on immunogold-labeled samples only to unambiguously identify isolated centrosome structures in the sample preparations. Centrosomes were varying in diameter between $0.48 \mu \mathrm{m}$ and $1.27 \mu \mathrm{m}(0.74 \mu \mathrm{m}$ on average, SD 0.15$)$ (Fig. 2a) and the distribution in centrosome size was continuous (Fig. 2a). The largest group $(20 \%)$ of centrosomes was between 0.71 and $0.78 \mu \mathrm{m}$ in size (Fig. 2b). However, we cannot discriminate whether the variations in size reflects an increase in size throughout the progression of the nuclear cycles nor can we rule out that the variations in centrosome size reflects the loss or gain of pericentriolar material throughout the centrosome purification procedure.

Distribution of pericentriolar and centriolar markers in the Drosophila centrosome

Subsequently, we investigated the distribution of a range of specific antigens of the pericentriolar matrix on the centrosome. In contrast, we used a marker for a centriolar antigen to relate centriole number to centrosome size.

We immunogold-labeled the centrosomes with antibodies against the following proteins: Asp, Cp60, Cp190, 3f/2-epitope, CNN, $\gamma$-tubulin, alpha-tubulin, MPM-2 and a centriole marker that has not been fully characterized on a molecular level. From these we show here four examples for the distribution of proteins that play important structural or functional roles. The isolated centrosomes were labeled with antibodies against gamma-tubulin that plays a direct role in microtubule nucleation (Fig. 3, row a-a") as well as for structurally important proteins such as CNN (Fig. 3, row b-b") and Asp (Fig. 3, row c- $\mathrm{c}^{\prime \prime}$ ). Finally, we also assayed the distribution of specific epitopes (MPM2 epitope) (Fig. 3, row d) that are phosphorylated through the mitotic kinases Polo and cdk1 (Logarinho and Sunkel 1996). Because of the limited three-dimensional nature of our whole mount assay we cannot discriminate the spatial distribution within the depth of the centrosome. All pericentriolar proteins showed within the limits of the indirect labeling approach (estimated to be $\pm 30 \mathrm{~nm}$ ) a homogenous distribution. This implied a regular spacing of structural as well as microtubule nucleating proteins in the centrosome (Fig. 3). The MPM2 antibody (Fig. 3, row d) gave the strongest labeling. This might be due to a high avidity of the antibody or, more likely, to the possibility that it labels multiple centrosomal proteins carrying the MPM2 phospho-epitopes. To test whether or not labeling of this antibody related to abundance of phospho-epitopes we treated the centrosomes in vitro with alkaline phosphatase. Subsequently, labeling was strongly reduced (Fig. 3, row $\mathrm{d}^{\prime \prime}$ ) confirming this assumption. Because this antibody labels essentially phospho-epitopes we cannot rule out that mitotic kinases in embryonic extract modify, that is, phosphorylate "non-mitotic" centrosomes throughout the isolation process. In addition to pericentriolar markers we used an anti-centriolar antibody that labeled either one or two distinct regions of the centrosomes. Of the 103 counted centrosomes $20 \%$ showed labeling of two 

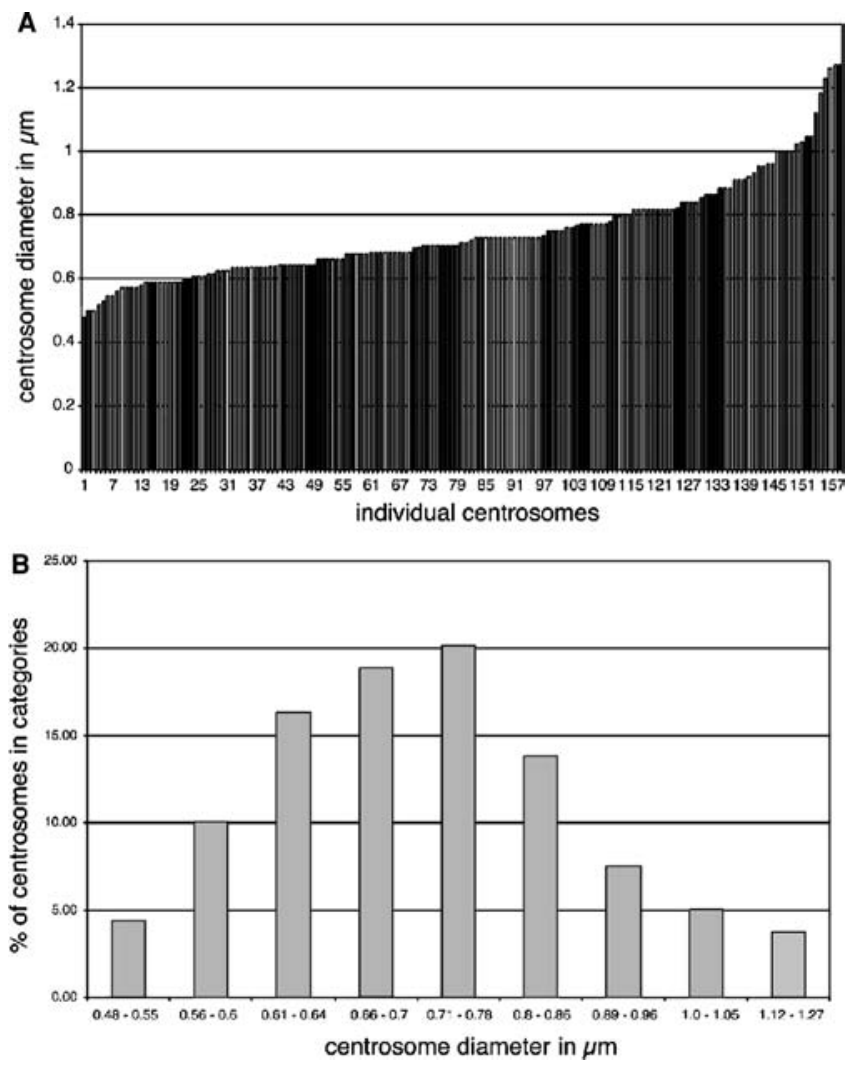

Fig. 2 Graphic representation of the size distribution of Drosophila centrosomes, measured on whole mount immunogold-labeled samples. a Individual centrosomes are represented by bars ( $x$-axis) whose length indicates the centrosome diameter in $\mu \mathrm{m}$ ( $y$-axis). Centrosomal diameter varies from 0.5 to $1.3 \mu \mathrm{m}$. The size variation has a small slope without major leaps. b The size distribution of centrosomes is normal. This graph shows the size distribution of centrosomes in categories. $x$-axis: size of centrosomes in $\mu \mathrm{m}, y$ axis: percent of centrosomes in a particular size category. The biggest (in number) group of centrosomes falls into the size category $0.71-0.78 \mu \mathrm{m}$

areas whereas the majority $(80 \%)$ contained only one labeled area (Fig. 3, row e). Since in the early Drosophila embryo centriole duplications occur in late mitosis (Callaini and Riparbelli 1990), this marker allowed us to correlate the size of centrosomes to the number of centrioles-like-structures stained. Accordingly, labeling of two centrioles was restricted to centrosomes that were between 0.8 and $1.3 \mu \mathrm{m}$ in diameter while the majority of centrosomes that display only a single labeled area were below $0.8 \mu \mathrm{m}$ in diameter. This indicates that the size of centrosomes correlates with the number of centrioles.

Analysis of the Drosophila centrosome in vitro microtubule nucleation capacity through immunofluorescence, SEM and TEM microscopy

The function of the centrosome was assayed in vitro by incubation with phosphocellulose-purified tubulin resulting in the formation of focused, aster-like micro- tubule arrays. The samples were then fixed and processed for immunofluorescence microscopy using antialpha-tubulin antibodies (Fig. 4a). Immunofluorescence microscopy has the benefit in this case to be a rapid functional assay. However, through the limitation in the resolution of the light microscope it cannot distinguish between single microtubules and microtubule bundles, especially in centrosome asters of very high microtubule density (Fig. 4a). Even more at the focus of the microtubules, which appears very bright in the fluorescence microscope, very little detail can be distinguished.

Scanning electron microcopy (SEM) (Fig. 4b, c) as well as transmission electron microscopy (TEM) (Fig. 4d, e) offers very good resolution of the microtubules that are around $25 \mathrm{~nm}$ in diameter. The SEM gives a three-dimensional representation of the microtubule asters and even permits to visualize single microtubules at the focal center of the asters (Fig. 4b, c). The TEM technique allows to visualize more fine details and also to quantify the number of microtubules nucleated by the centrosome (Fig. 4d, e).

Using TEM and SEM the number of microtubules that are originating at the centrosomes can be quantified in the periphery of individual asters. Indeed, both techniques show that the number of microtubules lies in a range from about 50 to around 300 microtubules measured on single non-overlapping asters. The graphical representation of microtubules nucleated per aster (Fig. 5a, b) has a steep slope indicating the sixfold difference in microtubule nucleation between the smallest and largest asters both in the TEM and the SEM experiments (TEM visualized asters, $n=107$; SEM visualized asters, $n=104)$. These data show that comparable results are obtained with the two different techniques. The size follows a normal distribution (Fig. 5c). The largest group of asters (16.7\%; SD 0.6) nucleate between 175 and 210 microtubules (Fig. 5c). The correlation of microtubule number nucleated to centrosome size was achieved using our negative staining technique to recognize the outline of the centrosome within the focus of microtubules directly on the EM negative. Consequently, these experiments could confirm the hypothesis that large centrosomes nucleate most microtubules. Hence, the majority $(48 \%)$ of centrosomes with a diameter between 0.5 and $1.2 \mu \mathrm{m}$ nucleated between 150 and 250 microtubules. More than 250 microtubules were nucleated by the fraction $(29 \%)$ of centrosomes with a diameter of $1.2 \mu \mathrm{m}$ and more.

\section{Discussion}

The duplication of the Drosophila centrosomes is synchronized with the rapid nuclear division cycles in the early embryo lasting only about $8-10 \mathrm{~min}$. The high mitotic activity of these centrosomes is reflected both in the abundant pericentriolar material as well as in the "minimalistic" centriole, that in early embryonic stages is neither composed of triplet microtubules nor carrying 

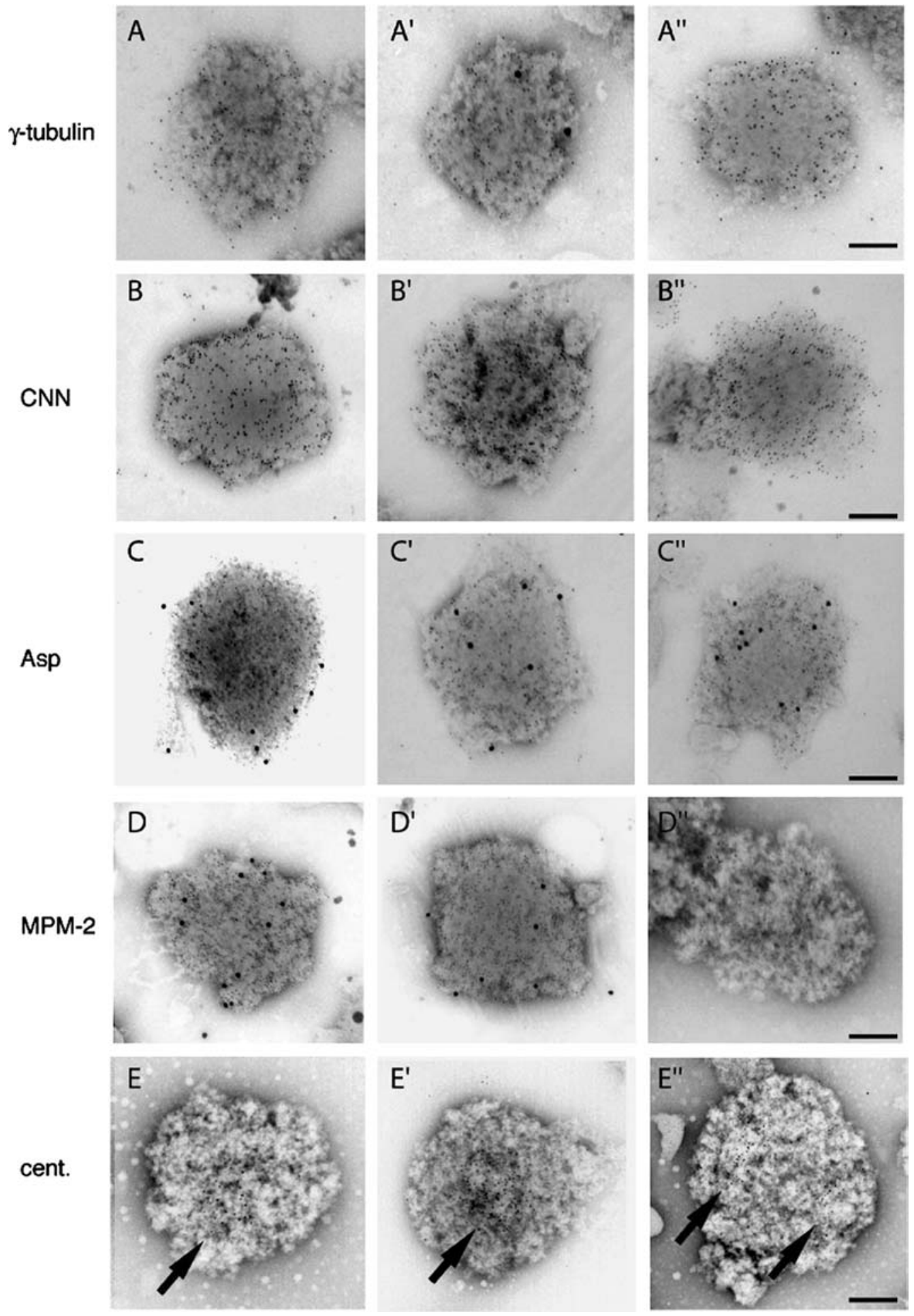

Fig. 3 Distribution of centrosomal antigens investigated on wholemount preparations of centrosomes using immuno-gold labeling and negative staining techniques. The localization of proteins with different functions was examined: $\gamma$-tubulin which is involved in

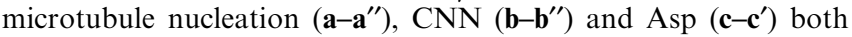
likely to be involved in the structural maintenance of the centrosome, and the generic phosphoeptiope antigen marked by the MPM2 antibody (d). The distribution of all peri-centriolar proteins is homogeneous and does not reveal any subdomain organization. The highest density of labeling is displayed by the MPM2 antibody (d, $\left.\mathbf{d}^{\prime}\right)$ indicating either a higher avidity of the antibody or a truly higher abundance of the eptiopes in the centrosome (bars in all images $=0.25 \mu \mathrm{m}$ ). The labeling with the MPM-2 antibody is strongly reduced in alkaline phophatase treated centrosomes $\left(\mathbf{d}^{\prime \prime}\right)$ demonstrating the dependency of labeling on phosphoepitopes. e In contrast to the anti-pericentriolar antibodies the anti-centriole marker labels a distinct domain in the central region of the centrosome (e). The majority of centrosomes show a single cluster (e; $\mathbf{e}^{\prime}$ ) of gold labeling (arrow), while about $20 \%$ of the centrosomes show labeling in two distinct clusters of gold particles, marking stages of the centrosomes containing two centriole structures 
the typical distal or subdistal appendages. These are acquired at later stages of development, for example, in differentiated cells such as in spermatocytes (reviewed by Gonzalez et al. 1998; Tates 1971). The centrosome of the Drosophila embryo has been described before in in situ electron microscopic studies (Callaini and Riparbelli 1990; Callaini et al. 1997; Debec et al. 1999; Stafstrom and Staehelin 1984; Vidwans et al. 1999). While these studies elucidated centriole structure and the centrosome duplication cycle, our work places the emphasis on the organization of centrosomal protein components and microtubule nucleation capacity of the isolated Drosophila centrosome. For this purpose we were using the technique of whole mount preparation of isolated centrosomes and in vitro nucleation of microtubules. Such an analysis of isolated centrosomes (as opposed to in situ) can be extremely informative in revealing structural features of this organelle in more detail (see the work of Chretien et al. 1997; Lange and Gull 1996b; Moritz et al. 1995a; Paintrand et al. 1992).

When compared to centrosomes isolated from other organisms, the overall appearance of the Drosophila centrosome resembles closest centrosomes from Spisula (Vogel et al. 1997). Both Drosophila and Spisula centrosomes have an abundant, amorphous pericentriolar material, although Spisula centrosomes are bigger (about two times) in overall size. Compared to centrosomes isolated from mammalian cells, Drosophila early embryonic centrosomes have an average size of about $0.75 \mu \mathrm{m}$ as compared to about $0.5 \mu \mathrm{m}$ for the typical mammalian centrosome. This size difference is due to the low amount of pericentriolar material in somatic mammalian cells and is illustrated by the fact that centrioles can easily be visualized in the mammalian centrosome (Komesli et al. 1989; Lange and Gull 1996b; Paintrand et al. 1992) also by whole mount techniques while the centriole from the early Drosophila embryo (as shown in our data) is almost impossible to discern concealed by the abundant pericentriolar material (compare also to TEM images of sections of isolated Drosophila centrosomes in Lange et al. (2000) and Moritz et al. (1995a).

Debec et al. (1999) characterized Drosophila early embryo centrosomes in situ through energy filtering TEM and observed in interphase centrosomes, structurally compact pericentriolar material that displayed long protrusion nucleating relatively few microtubules. In contrast mitotic centrosomes had a less dense pericentriolar material that nucleated more microtubules. In this respect it is intriguing to note that the density of pericentriolar material of isolated centrosomes without microtubules is higher than the density of centrosomes nucleating microtubules (Moritz et al. 1995a). This phenomenon might be reflected in the more compact pericentriolar material that has been described by Debec et al. (1999) nucleating less microtubules. In our electron microscope analysis the isolated centrosomes surface appearance was rough and fibrillar with relative small protrusions. We did not observe long protrusions of pericentriolar material, in agreement with the likely mitotic nature of centrosomes, but cannot rule out that structures might have been detached throughout the centrosome isolation procedure.

We interpret the abundant pericentriolar material together with the high number of nucleated microtubules as a marker of mitotic centrosomes (Khodjakov and Rieder 1999; Rieder and Borisy 1982; Vorobjev and Chentsov YuS 1982). This is also concomitant with the presence of MPM2 phosphoepitopes (Logarinho and Sunkel 1998) also detected in most of our centrosomes (see also Fig. 3, row d). The relatively high MTOC activity of Drosophila centrosomes becomes apparent when compared to in vitro nucleation experiments with mammalian centrosomes isolated from KE37 cells or calf thymus. The mammalian centrosomes in these experiments (Tournier et al. 1999; Lange et al. unpublished results) form much smaller microtubule asters carrying less microtubules.

This is in agreement with the fact that centrosomes isolated from mammalian somatic cells have predominantly the characteristics of centrosomes in interphase cells resulting from a mitotic index of only $4 \%$, for example, in exponentially growing Chinese hamster ovary (CHO) cells (Kuriyama 1984).

Indeed, when comparing the microtubule nucleation activity between isolated centrosomes from mammalian mitotic versus interphase cells (Kuriyama 1984; Kuriyama and Borisy 1981) microtubule nucleation activity in mitosis is five times higher. Centrosomes isolated from interphase $\mathrm{CHO}$ cells nucleate about 20-40 microtubules, mitotic centrosomes nucleate about 140-170 microtubules (Kuriyama and Borisy 1981) or, in a second study, 200-250 microtubules are nucleated from mitotic centrosomes under conditions of tubulin saturation (Kuriyama 1984). The fivefold increase corresponds also to data obtained from Xenopus egg extracts. In vitro experiments showed that the number of microtubules increased three to five times (from about 50 to more than 200 microtubules per centrosome) in egg extracts that had been mitotically arrested with cytostatic factors (Ohta et al. 1993).

Importantly, the number of microtubules nucleated depends on the concentration of tubulin used (Kuriyama 1984; Mitchison and Kirschner 1984). Moreover, the fact that the number of microtubules nucleated from a centrosome had a threshold indicates that the number of nucleation sites on the centrosome has been saturated (Mitchison and Kirschner 1984). Obviously, this consideration is significant for the determination of the maximum number of microtubule initiation sites on a centrosome. In our experiments, the number of nucleated microtubules varies between 50 and 300 per centrosome under saturating tubulin concentrations. This is consistent with a sixfold variation in the number of microtubules correlating to the variation of the size of isolated centrosomes. Thus, the capacity of centrosomes to nucleate microtubules is related to their size.

We calculated the relationship between centrosome surface, size and microtubules nucleated to assess whe- 

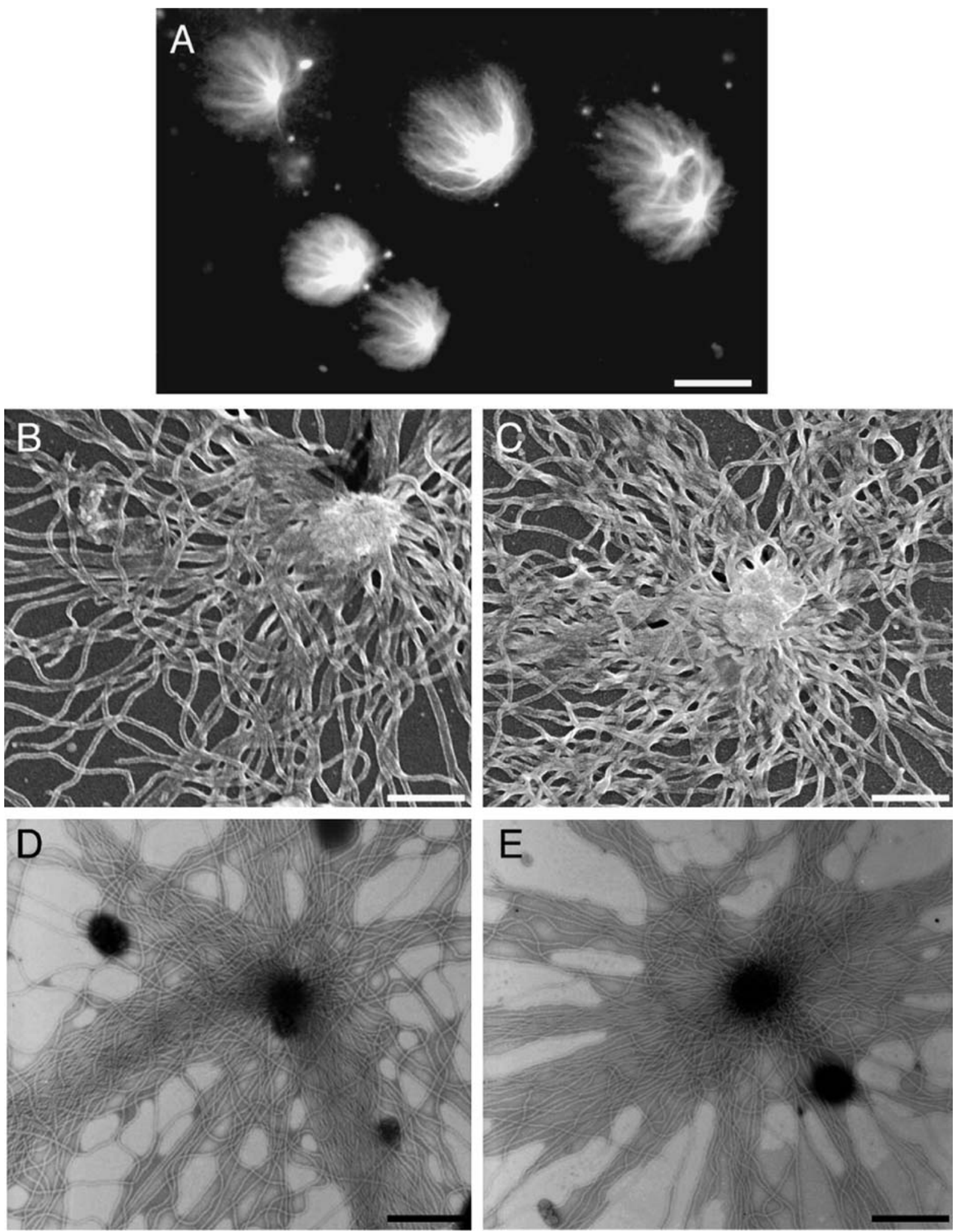

Fig. 4 Microtubule nucleation from isolated centrosomes with phosphocellulose purified tubulin, visualized by immunofluorescence microscopy (a), SEM (b, c) and TEM contrasted with goldthioglucose (d, e). a Six microtubule asters visualized by immunofluorescence microscopy with an anti-alpha-tubulin antibody; the density of the microtubules filaments is too high to

permit quantification of number of microtubules nucleated by each individual aster $(\mathrm{bar}=12 \mu \mathrm{m})$. $\mathbf{b}$, $\mathbf{c}$ SEM reveals fine details of the microtubule asters, resolving even microtubules at the center of the aster (in $\mathbf{b}$, bar $=0.5 \mu \mathrm{m}$; in $\mathbf{c}$, bar $=0.7 \mu \mathrm{m}$ ). d, e TEM resolves individual microtubules but the center of the aster appears dark through the accumulation of contrasting stain $(\mathrm{bar}=1.5 \mu \mathrm{m})$ 


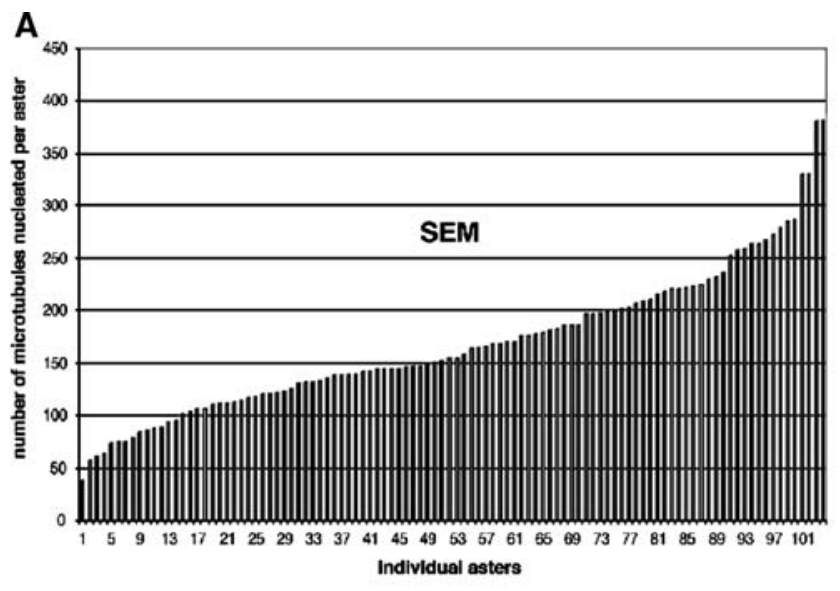

B

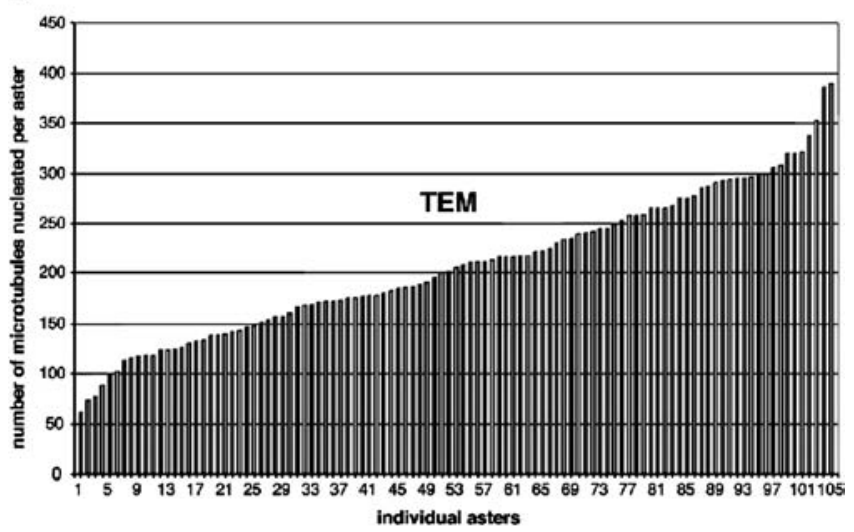

C

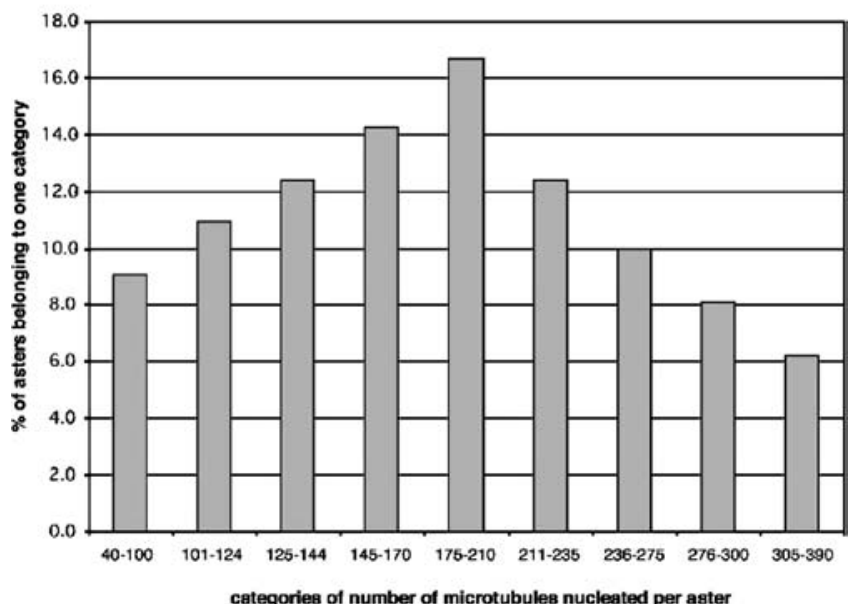

Fig. 5 Quantification of the number of microtubules nucleated per individual aster, visualized by a SEM or b TEM techniques. c The distribution of number of microtubules nucleated per aster is normal with the largest group (20\%) nucleating 175-210 microtubules per aster. The difference between small asters (nucleating approximately 50 microtubules) and big asters (nucleating approximately 300 microtubules) is about sixfold whether quantified by SEM or by TEM

ther a larger number of microtubules is correlated to a larger centrosomal surface supporting microtubule nucleation. Assuming a hypothetical spherical centrosome the surface area varies between 0.8 and $5 \mu \mathrm{m}^{2}$ corresponding to about sixfold increase of microtubule nucleation between small and large asters. The size variation of the centrosome is correlating with the number of microtubule nucleated from the larger, possibly late, mitotic centrosome. This is in agreement with the facts that the large centrosomes in our preparations contain two centrioles. In the Drosophila early embryo the duplicated centrioles separate in telophase (Callaini and Riparbelli 1990).

We propose that the number of microtubules nucleated is not directly limited by the surface of the centrosome but rather by the number of microtubule nucleation sites available. This hypothesis is in accordance with the fact that the minus ends of microtubules are distributed throughout the pericentriolar matrix (Moritz et al. 1995a) rather than only in the periphery of the centrosomes. This was suggested through automated electron tomography of in vitro microtubule nucleation experiments with isolated centrosomes.

One of the initial questions we raised was how many active gamma-tubulin ring complexes ( $\gamma$-TUPC) are to be expected in a (Drosophila) centrosome in vitro. Estimated from our measurements we expect isolated centrosomes to have between at least 50 and about 300 active $\gamma$-TuRCs to initiate microtubule nucleation. Wiese and Zheng (2000) propose that nucleation of a single microtubule end is achieved by $13 \gamma$-tubulin proteins in the $\gamma$-tubulin ring complex. Hence, we estimate that a Drosophila centrosome contains between 600 and 4000 active $\gamma$-tubulin subunits. Finally, we show that the whole mount electron microscopy technique is well suited to assess the centrosome in overall structural appearance, antigen distribution and to quantify its functional capacity to nucleate microtubules.

In the future a more complete molecular characterization of the centrosome will allow us to elucidate the precise three-dimensional organization and distribution of regulatory proteins, structural proteins or components that are directly involved in microtubule nucleation. Such a structural model of the centrosome will provide a better understanding on how its individual components and protein complexes might act together and how centrosome function and duplication are regulated. Previously, the detailed molecular and structural analysis of the yeast spindle pole, a functional homologue to the animal centrosome, has provided us with a wealth of information on how this organelle can function on a cellular level (Adams and Kilmartin 2000).

Acknowledgments We are grateful to T. Kaufman, D. Glover and S. Llamazares for antibody probes and to all members of the González laboratory and to A. Ploubidou for support and discussions. B. Lange was funded through a grant from the European Union, the Senatsverwaltung für Wissenschaft, Forschung und Kultur Berlin (Ultra-Structure Network), BMBF (NGFN2, SMP Protein). The González laboratory was supported by grants RTN-HPRNCT-2002-00260 COMBIO 503568, SAF 2003-07620 and FMMMA. Work in the Herzog laboratory was supported by the Deutsche Forschungsgemeinschaft (Research Group "Keratinocytes-Proliferation and Differentiation in the Epidermis"), 
the Fonds der Chemischen Industrie and the Bonner Forum Biomedizin.

\section{References}

Adams IR, Kilmartin JV (2000) Spindle pole body duplication: a model for centrosome duplication? Trends Cell Biol 10:329-335

Bornens M (2002) Centrosome composition and microtubule anchoring mechanisms. Curr Opin Cell Biol 14:25-34

Callaini G, Riparbelli MG (1990) Centriole and centrosome cycle in the early Drosophila embryo. J Cell Sci 97:539-543

Callaini G, Whitfield WG, Riparbelli MG (1997) Centriole and centrosome dynamics during the embryonic cell cycles that follow the formation of the cellular blastoderm in Drosophila. Exp Cell Res 234:183-190

Chretien D, Buendia B, Fuller SD, Karsenti E (1997) Reconstruction of the centrosome cycle from cryoelectron micrographs. J Struct Biol 120:117-133; doi: 10.1006/jsbi.1997.3928

Debec A, Marcaillou C, Bobinnec Y, Borot C (1999) The centrosome cycle in syncytial Drosophila embryos analyzed by energy filtering transmission electron microscopy. Biol Cell 91:379-391

Dictenberg JB, Zimmerman W, Sparks CA, Young A, Vidair C, Zheng Y, Carrington Fay FS, Doxsey SJ (1998) Pericentrin and gamma-tubulin form a protein complex and are organized into a novel lattice at the centrosome. J Cell Biol 141:163-174

Doxsey S (2001) Re-evaluating centrosome function. Nat Rev Mol Cell Biol 2:688-698

Evans L, Mitchison T, Kirschner M (1985) Influence of the centrosome on the structure of nucleated microtubules. J Cell Biol 100:1185-1191

Gonzalez C, Tavosanis G, Mollinari C (1998) Centrosomes and microtubule organisation during Drosophila development. J Cell Sci 111:2697-2706

Keating TJ, Borisy GG (2000) Immunostructural evidence for the template mechanism of microtubule nucleation. Nat Cell Biol 2:352-357

Kellogg DR, Moritz M, Alberts BM (1994) The centrosome and cellular organization. Annu Rev Biochem 63:639-674

Khodjakov A, Rieder CL (1999) The sudden recruitment of gamma-tubulin to the centrosome at the onset of mitosis and its dynamic exchange throughout the cell cycle, do not require microtubules. J Cell Biol 146:585-596

Komesli S, Tournier F, Paintrand M, Margolis RL, Job D, Bornens M (1989) Mass isolation of calf thymus centrosomes, identification of a specific configuration. J Cell Biol 109:2869-2878

Kuriyama R (1984) Activity and stability of centrosomes in Chinese hamster ovary cells in nucleation of microtubules in vitro. $\mathbf{J}$ Cell Sci 66:277-295

Kuriyama R, Borisy GG (1981) Microtubule-nucleating activity of centrosomes in Chinese hamster ovary cells is independent of the centriole cycle but coupled to the mitotic cycle. J Cell Biol 91:822-826

Lange BMH, Gull K (1996a) Structure and function of the centriole in animal cells - progress and questions. Trends Cell Biol 6:348-352

Lange BMH, Gull K (1996b) A structural study of isolated mammalian centrioles using negative staining electron microscopy. $\mathbf{J}$ Struct Biol 117:222-226; doi: 10.1006/jsbi.1996.0086

Lange BMH, Bachi A, Wilm M, Gonzalez C (2000) Hsp90 is a core centrosomal component and is required at different stages of the centrosome cycle in Drosophila and vertebrates. EMBO J $19 \cdot 1252-1262$

Li K, Kaufman TC (1996) The homeotic target gene centrosomin encodes an essential centrosomal component. Cell 85:585-596

Logarinho E, Sunkel CE (1998) The Drosophila POLO kinase localises to multiple compartments of the mitotic apparatus and is required for the phosphorylation of MPM2 reactive epitopes. J Cell Sci 111:2897-2909

MacRae TH, Lange BMH, Gull K (1990) Production and characterization of monoclonal antibodies to the mammalian sperm cytoskeleton. Mol Reprod Dev 25:384-392

Marshall W (2001) Centrioles take center stage. Curr Biol 11:R487-R496

Mitchison T, Kirschner M (1984) Microtubule assembly nucleated by isolated centrosomes. Nature 312:232-237

Moritz M, Braunfeld MB, Fung JC, Sedat JW, Alberts BM, Agard DA (1995a) Three-dimensional structural characterization of centrosomes from early Drosophila embryos. J Cell Biol 130:1149-1159

Moritz M, Braunfeld MB, Sedat JW, Alberts B, Agard DA (1995b) Microtubule nucleation by gamma-tubulin-containing rings in the centrosome. Nature 378:638-640

Moritz M, Zheng Y, Alberts BM, Oegema K (1998) Recruitment of the gamma-tubulin ring complex to Drosophila salt-stripped centrosome scaffolds. J Cell Biol 142:775-786

Moritz M, Braunfeld MB, Guenebaut V, Heuser J, Agard DA (2000) Structure of the gamma-tubulin ring complex: a template for microtubule nucleation. Nat Cell Biol 2:365-370

Ohta K, Shiina N, Okumura E, Hisanaga S, Kishimoto T, Endo S, Gotoh Y, Nishida E, Sakai H (1993) Microtubule nucleating activity of centrosomes in cell-free extracts from Xenopus eggs: involvement of phosphorylation and accumulation of pericentriolar material. J Cell Sci 104:125-137

Paintrand M, Moudjou M, Delacroix H, Bornens M (1992) Centrosome organization and centriole architecture: their sensitivity to divalent cations. J Struct Biol 108:107-128

Rieder CL, Borisy GG (1982) The centrosome cycle in in PtK2 cells: asymmetric distribution and structural changes in the pericentriolar material. Biol Cell 44:117-137

Rothwell WF, Sullivan W (2000) The centrosome in early Drosophila embryogenesis. Curr Top Dev Biol 49:409-447

Saunders RD, Avides MC, Howard T, Gonzalez C, Glover DM (1997) The Drosophila gene abnormal spindle encodes a novel microtubule-associated protein that associates with the polar regions of the mitotic spindle. J Cell Biol 137:881-890

Schnackenberg BJ, Khodjakov A, Rieder CL, Palazzo RE (1998) The disassembly and reassembly of functional centrosomes in vitro. Proc Natl Acad Sci USA 95:9295-9300

Stafstrom JP, Staehelin LA (1984) Dynamics of the nuclear envelope and of nuclear pore complexes during mitosis in the Drosophila embryo. Eur J Cell Biol 34:179-189

Tates AD (1971) Cytodifferentiation during spermatogenesis in Drosophila melanogaster. An electron microscope study. Rijksuniversiteit Leiden, Leiden

Tournier F, Bobinnec Y, Debec A, Santamaria P, Bornens M (1999) Drosophila centrosomes are unable to trigger parthenogenetic development of Xenopus eggs. Biol Cell 91:99-108

Vidwans SJ, Wong ML, O'Farrell PH (1999) Mitotic regulators govern progress through steps in the centrosome duplication cycle. J Cell Biol 147:1371-1378

Vogel JM, Stearns T, Rieder CL, Palazzo RE (1997) Centrosomes isolated from Spisula solidissima oocytes contain rings and an unusual stoichiometric ratio of alpha/beta tubulin. J Cell Biol 137:193-202

Vorobjev IA, Chentsov YS (1980) The ultrastructure of centriole in mammalian tissue culture cells. Cell Biol Int Rep 4:1037-1044

Vorobjev IA, Chentsov YuS (1982) Centrioles in the cell cycle I. Epithelial cells. J Cell Biol 93:938-949

Vorobjev IA, Nadezhdina ES (1987) The centrosome and its role in the organization of microtubules. Int Rev Cytol 106:227293

Wiese C, Zheng Y (2000) A new function for the gamma-tubulin ring complex as a microtubule minus-end cap. Nat Cell Biol $2: 358-364$ 